Research article

\title{
BDNF and serum S100B levels according the spectrum of structural pathology in chronic pain patients
}

\author{
Luciana Cadore Stefani $^{1,2, *}$, Fabricio M. Leite ${ }^{1}$, Maria da Graça L. Tarragó ${ }^{3}$, Simone A. Zanette ${ }^{3}$, \\ Andressa de Souza ${ }^{4}$, Stela M. Castro ${ }^{5,6}$, Wolnei Caumo ${ }^{7,8}$ \\ ${ }^{1}$ Postgraduate Program in Medical Sciences, School of Medicine, Universidade Federal do Rio Grande do Sul (UFRGS), Porto Alegre, RS, Brazil \\ ${ }^{2}$ Adjunt professor at Department of Surgery, School of Medicine, UFRGS, Porto Alegre, RS, Brazil \\ ${ }^{3}$ Department of Surgery, School of Medicine, UFRGS, Porto Alegre, RS, Brazil \\ ${ }^{4}$ Postgraduate Program in Health and Human Development, La Salle University Center, Canoas, RS, Brazil \\ ${ }^{5}$ Postgraduate Program in Epidemiology, School of Medicine-Universidade Federal do Rio Grande do Sul (UFRGS), Porto Alegre, RS, Brazil \\ ${ }^{6}$ Satistical Department, Mathematical and Statistical Institute, UFRGS, Porto Alegre, RS, Brazil \\ ${ }^{7}$ Postgraduate Program in Medical Sciences, School of Medicine, Universidade Federal do Rio Grande do Sul (UFRGS), Porto Alegre, RS, Brazil \\ ${ }^{8}$ Department of Surgery, School of Medicine, UFRGS, Laboratory of Pain and Neuromodulation, HCPA, Porto Alegre, RS, Brazil
}

\section{A R T I C L E I N F O}

\section{Keywords:}

Brain-derived neurotrophic factor

S100B

Central sensitization syndrome

Osteoarthritis

Fibromyalgia

Tension headache

\begin{abstract}
A B S T R A C T
Central sensitivity syndrome (CSS) consists of adaptive pathophysiological changes associated with neuroplasticity in some chronic pain disorders. It could be grouped in two main conceptual conditions: one includes those chronic pain patients without overt structural pathology such as fibromyalgia, and the other subgroup includes conditions with recognizable structural abnormalities, both somatic (osteoarthritis) and visceral (endometriosis). In order to understand the role of neuromodulators in CCS we aim to determine whether brain-derived neurotrophic factor (BDNF) and S100B are associated to specific chronic pain disorders. Serum BDNF and S100B were measured in chronic pain women with different diagnosis: 88 with osteoarthritis, 36 with endometriosis, 117 with fibromyalgia, 33 with chronic tension type headache and in 41 healthy controls. ANCOVA analysis followed by heteroscedasticity-consistent covariance matrix was performed to evaluate BDNF and S100B levels, adjusted for depression severity, pain levels and use of analgesics according different pathologies. Serum BDNF concentrations were higher and not different in patients with fibromyalgia and headache, the CSS group without structural pathology. In contrast, the concentrations of S100B were higher in patients with osteoarthritis and endometriosis, in comparison to controls, fibromyalgia and tensional headache patients. This study supports the hypothesis that BDNF and S100B neuromodulators present different serum levels according to the background disease associated to the chronic pain. These have the potential to be studied as markers of active disease or treatment evolution.
\end{abstract}

\section{Introduction}

Chronic pain represents one of the main causes of incapacity and suffering in adult life. Central sensitivity syndrome (CSS) consists of adaptive pathophysiological changes mediated by the central nervous system (CNS) associated with neuroplasticity in some chronic pain disorders [1]. It depicts an enlargement of the neurons and circuits functions of the nociceptive pathway caused by increased excitability and synaptic efficacy, as well as the reduction of inhibition. It includes symptoms as psychological distress, sleeping disorders, fatigue, pain, allodynia, hyperalgesia, expansion of the receptive field, prolonged electrophysiological discharge, and an after-stimulus unpleasant quality of the pain $[1,2]$.

The CSS could be grouped in two main conceptual conditions: one includes those without overt structural pathology, such as fibromyalgia, myofascial pain syndrome, and chronic tensional type headache (CTTH). The other subgroup includes conditions with recognizable structural abnormalities, both somatic (osteoarthritis) and visceral (endometriosis) [3,4]. Identifying the chronic pain patients with CSS helps to design the treatment, to agree to the prognostic and to get the patients and their families involved.

To delineate the profile of central sensitization conditions, some objective testing can be done, including functional magnetic resonance and cerebral evoked potential [5]. Also, the physiology of CSS involves

\footnotetext{
* Corresponding author.

E-mail address: lpstefani@hcpa.edu.br (L.C. Stefani).
} 
activation of several neurotransmitters and neuromodulators acting along pain pathways which could be used in a more accessible manner (blood sampling) to help the CSS identification [5].

Brain-derived neurotrophic factor (BDNF) is a remarkable marker and modulator of neuronal activity and $N$-methyl-D-aspartate (NMDA) receptor dependent neuronal plasticity in pain transmission pathways [6]. In animals, when neutralizing BDNF by anti-BDNF antibody, mechanical allodynia [7] and thermal hyperalgesia were relieved [8]. In humans, our group has demonstrated higher levels of BDNF in patients with fibromyalgia, headache, and myofascial pain [3]. Furthermore, BDNF serum levels were significantly correlated with decreased inhibitory system as assessed by conditioned pain modulation [9] and with the reduction of pressure pain thresholds in fibromyalgia [10].

$\mathrm{S} 100 \mathrm{~B}$ is a calcium-binding protein associated to the upregulation of other mediators expression such as Interleukin 1-Beta and Tumor Necrosis Factor (TNF) and to the activation of the nuclear factor kappalight-chain-enhancer of activated B cells (NF-kB) in microglia and astrocytes [11]. In animal models, S100B was associated to hyperalgesia [12], and in humans with fibromyalgia; it was associated to reduced pain thresholds in pressure pain testing [10].

Considering the common mechanisms shared by some diseases with peripheral and central mechanisms associated to CSS, it is reasonable to hypothesize that BDNF and S100B serum levels may have different courses according to the disease associated to chronic pain and central sensitization diagnosis.

\section{Methods}

\subsection{Design overview, settings, and participants}

This is a cross-sectional study in which the final sample data was pooled from five clinical trials from September 2012 to March 2013 at the Pain and Neuromodulation Research Group of Hospital de Clínicas de Porto Alegre (HCPA). All the trials were approved by the HCPA Ethics Committee and the informed written consent was obtained from all subjects. The studies involved women with chronic pain conditions such as fibromyalgia (NCT02041455; NCT01804097), osteoarthritis (NCT01747070; NCT01855958), endometriosis (NCT02161302) and CTTH (NCT01954277). Details of the inclusion of each study can be seen in Fig. S1 (Supplementary material). Patients were recruited from the institutional chronic pain clinic or from other clinic units and media advertisement. Patients who didn't understand Brazilian Portuguese or who presented cancer, uterine fibroids, ovarian cysts, inflammatory pelvic disease, pregnancy, and kidney or hepatic insufficiency were excluded. Each clinical trial had strict inclusion criteria and the diagnoses were confirmed by a doctor with more than 15 years of experience in chronic pain. The diagnosis was determined by clinical complaints and physical examination, as well as current and past medication, medical and psychiatric care. Within it were patients who had experienced pain both scored in the visual analog scale (VAS) as $>40 \mathrm{~mm}$ (i.e., moderate or severe pain - which was an inclusion criteria shared by the clinical trials used) [13] and that lasted $>3$ months, associated with functional disability. This was assessed inquiring whether the disease had troubled patients, in the last 3 months, with work; enjoyable activities; responsibilities at home; relationships; personal goals and thinking clearly. For inclusion, patients had to have at least one positive answer.

Trials details criteria are summarized as follows:

1 Osteoarthritis: was confirmed by clinical and radiographic criteria of the American College of Rheumatology [14] and by the Western Ontario and McMaster Universities Osteoarthritis Index (WOMAC) [15]. Patients with a disability score on the WOMAC $\geq 21$ were included.

2 Fibromyalgia: diagnosis was confirmed by the 2010 American College of Rheumatology criteria [16].
3 Endometriosis: was confirmed by laparoscopy associated with pelvic pain and/or dyspareunia of moderate to severe intensity (pain level $>4$ on the VAS) [13] lasting $>6$ months.

4 CTTH: diagnosis was based on criteria from the International Classification of Headache Disorders [17]. Participants had to experience headaches with following symptoms for more than 180 days in the last year: pain lasting hours to days; bilateral, pressing or tightening quality; mild or moderate intensity; and not aggravated by routine physical activities. A headache specialist confirmed the diagnosis.

5 Volunteers: Pain-free control volunteers were invited from the community using media advertisement. Painful condition; analgesics or corticosteroids use; rheumatologic, psychiatric, or neurological disorder; alcohol abuse or psychotropic substances use during the 6 months previous to the screening; or medications with effects on the CNS use were exclusion criteria.

\subsection{Serum neuroplasticity mediators' concentration}

Biological samples were collected early in the morning. All the material was obtained in vacutainer tubes without anticoagulant and centrifuged for $10 \mathrm{~min}$ at $4500 \mathrm{rpm}$ at $4^{\circ} \mathrm{C}$. The serum was frozen at $-80^{\circ} \mathrm{C}$ until further analyses. Serum mediators' concentrations were determined by using BDNF (Millipore, Missouri, USA, catalogue \#CYT306, kit's lower detection limit $=7.8 \mathrm{pg} / \mathrm{mL}$ ) and S100B (Millipore, Missouri, USA, catalogue \#EZHS100B-33 K, kit's lower detection limit $=2.7 \mathrm{pg} / \mathrm{mL}$ ) enzyme-linked immunosorbent assay kits (ELISA), according to the manufacturers' instructions.

\subsection{Potential confounding variables}

All psychological tests used had been validated for the Brazilian population. The patients' depressive symptoms were assessed using the Hamilton Depression Rating Scale (HDRS) [18] and were classified as mild if ranging from 11 to 17 ; and moderate to severe if $\geq 18$. OA patients' answered the Beck Depression Inventory (BDI) and the depressive symptoms were classified as mild if $\leq 18$ and as moderate to severe if $>18$ [19]. The number of doses used weekly within the last 3 months determined analgesic use.

\subsection{Statistical analyses}

Comparisons between continuous variables were performed using either ANOVA or Kruskal-Wallis tests according to the variables' distribution. To evaluate the supposition of ANOVA model we proceed to the residual analysis. Categorical variables were compared using the Chi-square or Fisher's exact test. ANCOVA models were adjusted for variables $\mathrm{S} 100 \mathrm{~B}$ and BDNF, aiming to identify significant difference between their means according the presence or absence of structural pathology.

Covariates that could interfere in these results were included in the model: pain severity, age, degree of depressive symptoms and use of psychotropic medications. Also another ANCOVA model was used to deeply explore the differences between the biomarkers' levels in each disease. The covariates were checked for their multicollinearity. The model was adjusted to deal with heteroscedasticity through the heteroscedasticity-consistent covariance matrix [20]. The alpha level was set at 0.05 . The data were analyzed using SAS Studio 9.4.

\section{Results}

A sample of 315 patients was allocated from six different studies conducted by the same research group. Three hundred eighty-two patients were excluded because they did not meet the inclusion criteria (VAS $<40 \mathrm{~mm}$ ). According to their underlying pathologies, chronic pain patients were divided into two groups considering the CSS with 
A

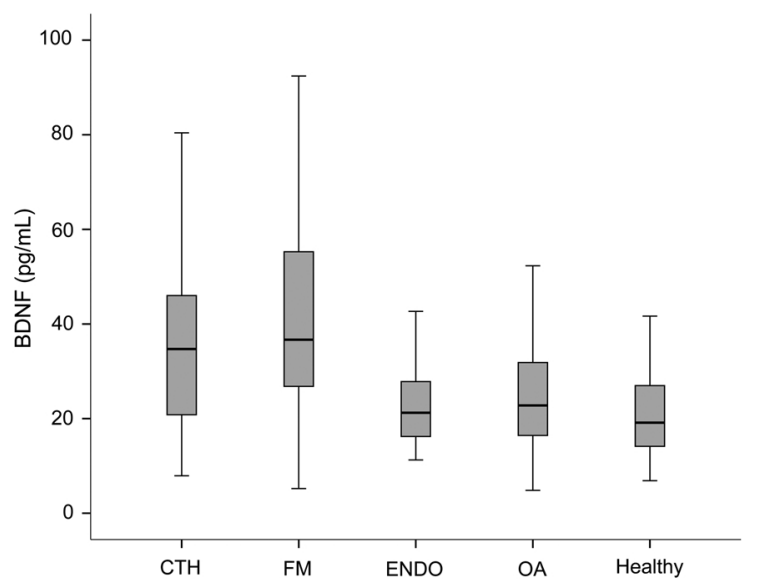

B

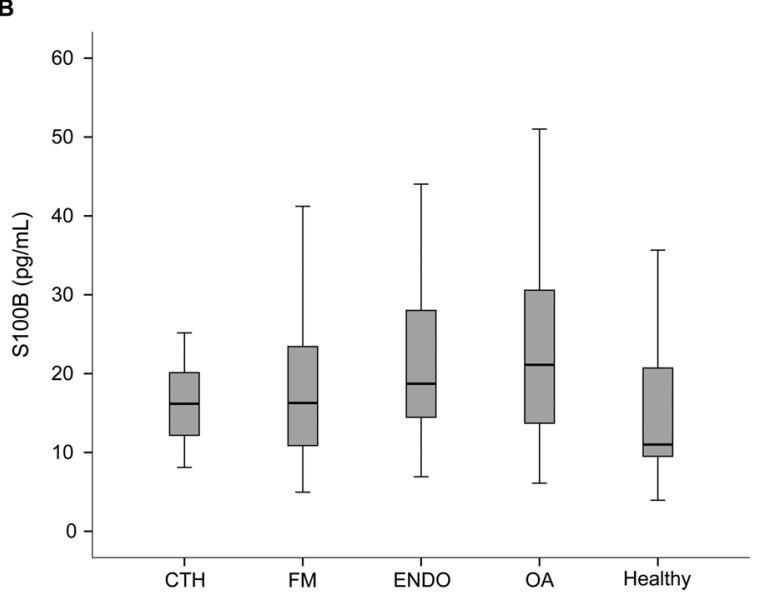

Fig. 1. Box plot of neuromodulators informing the spread and variability of BDNF (A) and S100B (B) data by multiple categories of diseases. The concentration of BDNF and S100B are in $\mathrm{pg} / \mathrm{mL}$. BDNF $=$ brain-derived neurotrophic factor. $\mathrm{CTH}=$ chronic tension headache. $\mathrm{ENDO}=$ endometriosis. $\mathrm{FM}=$ fibromyalgia. $\mathrm{OA}=$ osteoarthritis

persistent somatic/visceral nociceptive input (the presence of structural lesion) or its absence. Patients with osteoarthritis $(n=88)$ and endometriosis $(\mathrm{n}=36)$ were included in the group with structural pathology, and patients with fibromyalgia $(n=117)$ and tensional headache $(n=33)$ were grouped as absent structural lesion. For the non-patient comparison sample, 41 female volunteers were screened (Fig. S1 - Supplementary material).

Groups were classified with respect to age, body mass index, pain severity, depressive symptoms, and use of pain medications (Table S1 Supplementary material). The distribution of data value of BDNF and S100B according the different pathologies was illustrated by the box plot (Fig. 1).

Table 1 shows the result of serum BDNF and S100B concentration values according presence or absence of structural pathology, controlled for confounding factors such as age, degree of pain, depression or analgesic consumption. The kind of disease had a significant effect on BDNF, $\quad\left(\mathrm{F}_{2,303}=19.21 ; \mathrm{p}<0.01\right)$ and on S100B levels $\left(\mathrm{F}_{2,293}=11.70, \mathrm{p}<0.01\right)$.

Table 2 shows the result of ANCOVA model with heteroscedasticityconsistent covariance matrix used to compare the result of BDNF and S100B serum concentrations according to the different pathologies, controlled for confounding factors. The kind of chronic pain condition had a significant effect on BDNF, $\mathrm{F}_{4,301}=9.59$; $\mathrm{p}<0.01$ ) and on S100B levels, $\mathrm{F}_{2,293}=8.01 ; \mathrm{p}<0.01$. Table S2 (Supplementary material) shows the complete result of ANCOVA analysis.
Table 1

Serum neuroplasticity mediators' concentration according presence or absence of structural pathology. Values are expressed in $\mathrm{pg} / \mathrm{mL}$

\begin{tabular}{lllll}
\hline $\begin{array}{l}\text { Neuroplasticity } \\
\text { mediators }\end{array}$ & $\begin{array}{l}\text { Without } \\
\text { structural lesion }\end{array}$ & $\begin{array}{l}\text { With structural } \\
\text { lesion }\end{array}$ & Healthy & $\mathrm{p}$ \\
\hline BDNF levels $(\mathrm{pg} / \mathrm{mL})$ & $38.48^{\mathrm{a}}$ & $24.22^{\mathrm{b}}$ & $23.13^{\mathrm{b}}$ & $<0.01$ \\
S100B levels $(\mathrm{pg} / \mathrm{mL})$ & $18.22^{\mathrm{a}}$ & $22.64^{\mathrm{b}}$ & $14.34^{\mathrm{a}}$ & $<0.01$ \\
\hline
\end{tabular}

Differences were tested using ANCOVA. The variables included in the model were: moderate/severe depression, age, pain scale and use of pain medication. The groups sharing a letter are not significantly different. The model was adjusted to deal with heteroscedasticity through the heteroscedasticity-consistent covariance matrix.

BDNF was higher and not different in patients with fibromyalgia and headache, the CSS group without structural pathology, compared to osteoarthritis, endometriosis and healthy groups. In contrast, the concentrations of S100B were higher in patients with osteoarthritis and endometriosis, the CSS group with structural pathology, in comparison to controls, fibromyalgia and tensional headache patients.

\section{Discussion}

This study supports the hypothesis that BDNF and S100B neuromodulators present different serum levels according to the background disease associated to the chronic pain.

Our main find is the confirmation of BDNF increase in patients with CSS without structural pathology, as in fibromyalgia and chronic tension headaches. In these groups, BDNF serum level - controlled to other factors such as depression, pain levels, and the use analgesics - was significantly greater than in women who are healthy or affected by diseases with the presence of structural pathology, namely with persistent nociceptive stimulus.

Both fibromyalgia and chronic tension headache share common sensitization mechanisms as amplification of afferent signals and inadequate descendent inhibitory control [21] associated to a psychological chronic stress. In CTTH it has been demonstrated that continuous nociceptive input from pericranial myofascial tissues may induce supraspinal sensitization and thereby the chronification of headaches [22]. The psychological stress may cause a prolonged increase of muscle tone via the limbic system and also potentiate pain facilitation from the brain stem to the spinal dorsal horn [23].

In fibromyalgia there is a scarce evidence of structural pathology, that is, a non-nociceptive component related to the phenomenon of central sensitization. It is considered a disorder of the nociceptive processing with immunity system deregulation and inflammation [24]. In addition, affective changes and mood disorders are typically observed in both diseases. BDNF orchestrates mechanisms of neuronal plasticity and survival, holding the capacity to sensitize peripheral nociceptors and increasing excitability of second order neurons. It has already been shown that intact BDNF crosses the blood-brain-barrier by a high-capacity, saturable transport system [25]. Even though its serum levels in humans are affected by time of day of blood withdrawal, age, sex, urbanicity, smoking status and alcohol use, the effect sizes of these variables are generally small [26].

In pain processes, BDNF levels alter the descending nociceptive inhibition towards nociceptive facilitation in the brain. It is also an important facilitator of long-term potentiation (LTP), especially in regions involved in learning and memory, which may be related to the generation of pain memories in chronic pain patients [27]. Giving this multiplicity of actions, our results corroborate that pain associated to central sensitivity and to central nervous dysfunction has a biological substrate. In a previous study of our group we observed increased levels of BDNF and TNF- $\alpha$ in patients with CSS without structural pathology [3]. In addition, we demonstrated that serum BDNF may be involved in processes that mediate the disinhibition of motor cortex excitability, 
Table 2

Serum neuroplasticity mediators' concentration according to different conditions. Values are expressed in $\mathrm{pg} / \mathrm{mL}$.

\begin{tabular}{|c|c|c|c|c|c|c|}
\hline Neuroplasticity mediators & Pain-free controls $(n=17)$ & Osteoarthritis $(\mathrm{n}=86)$ & Endometriosis $(\mathrm{n}=36)$ & Fibromyalgia $(\mathrm{n}=79)$ & Headache $(\mathrm{n}=33)$ & $\mathrm{p}$ \\
\hline BDNF levels (pg/mL) & $22.85^{\mathrm{b}}$ & $24.85^{\mathrm{b}}$ & $23.71^{\mathrm{b}}$ & $38,60^{\mathrm{a}}$ & $37.22^{\mathrm{a}}$ & $<0.01$ \\
\hline S100B levels (pg/mL) & $11,23^{\mathrm{a}}$ & $23,99^{\mathrm{b}}$ & $20,27^{\mathrm{b}}$ & $19,40^{\mathrm{a}}$ & $14,82^{\mathrm{a}}$ & $<0.01$ \\
\hline
\end{tabular}

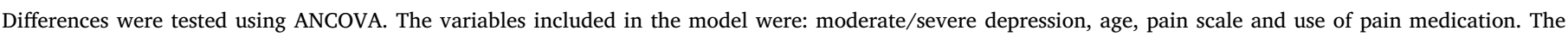

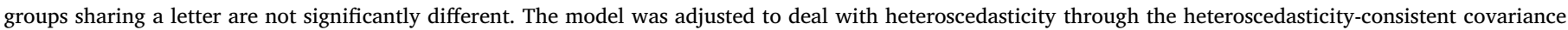
matrix.

along with the function of the descending inhibitory pain modulation system, independently of the physiopathology mechanism of musculoskeletal pain syndromes [9]. In patients with CTTH BDNF levels changed after pain modulation provided by electroacupuncture analgesia versus sham stimulation [28].

These findings are important in narrowing the gap between the patient's perception of their illness and their health care professional. Patients with 'unexplained' chronic musculoskeletal pain who are misinformed about pain, have low adherence for active treatments [29]. The objective serum biological marker could help reconceptualise pain before initiating the treatment. Also, this approach can prevent the patient from suffering about the scientific background of their pain. The early CSS diagnosis can help reduce the staff and resources invested in misleading and stressful tests, enabling efforts to be directed toward safer and effective interventions (e.g., psychotherapy, physical activity) [3].

Distinctively, while BDNF was predominantly higher in chronic pain patients without structural pathology, S100B levels were predominantly elevated in patients with CSS with structural pathology, represented by osteoarthritis and endometriosis. Osteoarthritis carries a strong nociceptive joint component associated to central sensitization in advanced stages. This central sensitization was demonstrated in a cross-sectional study on women with osteoarthritis in which the change in cortical plasticity was associated with less intracortical inhibition [30]. Several key mediators are particularly involved in the generation and maintenance of osteoarthritis pain, such as nerve growth factor (NGF), and cytokines [31].

Glia is thought to be involved in the propagation and modulation of acute and chronic painful stimuli, as well as in the neurotransmission and plastic changes. S100B protein is released from the glia after neuronal damage, for instance, after a head injury [12] or cardiopulmonary bypass. Furthermore, in a recent study with patients submitted to bilateral total knee arthroplasty, liquoric S100B decreased significantly one week after the operation, compared to the basal value [32].

Patients with endometriosis also have shown elevated basal levels of S100B in our sample. Endometrial lesions give rise to different types of pain, including visceral and myofascial nociception. Visceral nociception and peripheral sensitization are secondary to the direct innervation of endometrial injuries, but over time central sensitization creates a process for pain sustention that is independent of the initial pathology. Besides, the viscerosomatic convergence provides the means for pain referral to somatic structures and induces the muscle spasm reflex and the eventual formation of myofascial trigger points too. These trigger points, in turn, may serve as an extra source of nociceptive input, becoming a key component of chronic pelvic pain [33]. As far as we know, this is the first exploratory study that has shown increased S100B levels in women with moderate to severe chronic pelvic pain. Giving the complex pain physiopathology associated to endometriosis, it is reasonable to consider S100B as promising biomarker.

Our results should be interpreted in light of some limitations. First, neuroplasticity mediators are not specific for CSS, once these have demonstrated association in different scenarios other than pain-like mood or psychiatric disorders, inflammation, trauma, and even physical exercises [34]. BDNF and S100B could also be influenced by both pain severity and depression symptoms. These two factors differed between our samples and to address the potential confounding effect we included them as covariates in our analysis. Second, the division of CSS in absence or presence of structural pathology may be suboptimal. Granted the complexity of each syndrome and the presence of multiple mechanisms that could contribute to the final perception of pain, an approach to design the signature of each disease could be considered. Third, our sample is strictly made of females and the results related to BDNF could not be extrapolated to males. In an experimental acute pain model in volunteers, it was demonstrated that gender interferes in BDNF levels [35]. Also, the osteoarthritis group had significantly older age compared to other pathologies. Finally the study's cross-sectional nature allows associations and not any causality inference, which requires future investigations.

\section{Conclusions}

Findings from this study have shown increased BDNF levels in chronic pain patients with CSS in the absence of tissue injury. Otherwise, S100B had predominantly increased levels in chronic pain patients with tissue injury. Taking into consideration the overlapping symptoms and pathophysiological findings in some conditions, these biomarkers could be used individually as an objective profile validation of the CSS. Also, they have the potential to be studied as markers of either the active disease or treatment evolution.

\section{Contributors}

LCS and WC contributed with methodology, supervision, formal analysis, writing, editing, conception and interpretation of the work, and revised it critically. FML helped with methodology and data collection. MGLT and SAZ performed data collection and experimental analyses. AS helped with conceptualization and methodology. SMJC undertook data curation, software and statistical analyses. All authors approved the final version.

\section{Role of funding sources}

This work was supported by Research and Events Incentive Fund Hospital de Clínicas de Porto Alegre (FIPE-HCPA), Brazil. The funding agency did not have any role in the study design, analysis or manuscript preparation.

\section{Declarations of interest}

None.

\section{Acknowledgements}

The authors are thankful for the support provided by FIPE-HCPA.

\section{Appendix A. Supplementary data}

Supplementary material related to this article can be found, in the online version, at doi:https://doi.org/10.1016/j.neulet.2019.05.021. 


\section{References}

[1] M.B. Yunus, Central sensitivity syndromes: a new paradigm and group nosology for fibromyalgia and overlapping conditions, and the related issue of disease versus illness, Semin. Arthritis Rheum. 37 (2008) 339-352.

[2] R. Staud, M.E. Rodriguez, Mechanisms of disease: pain in fibromyalgia syndrome, Nat. Rev. Rheumatol. 2 (2006) 90-98.

[3] A. Deitos, J.A. Dussán-Sarria, A. de Souza, L. Medeiros, M. da Graça Tarragô, F. Sehn, M. Chassot, S. Zanette, A. Schwertner, F. Fregni, I.L.S. Torres, W. Caumo, Clinical value of serum neuroplasticity mediators in identifying the central sensitivity syndrome in patients with chronic pain with and without structural pathology, Clin. J. Pain 31 (2015) 959-967.

[4] S.E. Gwilym, J.R. Keltner, C.E. Warnaby, A.J. Carr, B. Chizh, I. Chessell, I. Tracey, Psychophysical and functional imaging evidence supporting the presence of central sensitization in a cohort of osteoarthritis patients, Arthritis Care Res. 61 (2009) 1226-1234.

[5] M.B. Yunus, Fibromyalgia and overlapping disorders: the unifying concept of central sensitivity syndromes, Semin. Arthritis Rheum. 36 (2007) 339-356.

[6] R.C. Malenka, M.F. Bear, LTP and LTD: an embarrassment of riches, Neuron 44 (2004) 5-21.

[7] X.F. Zhou, Y.S. Deng, C.J. Xian, J.H. Zhong, Neurotrophins from dorsal root ganglia trigger allodynia after spinal nerve injury in rats, Eur. J. Neurosci. 12 (2000) 100-105.

[8] T. Fukuoka, E. Kondo, Y. Dai, N. Hashimoto, K. Noguchi, Brain-derived neurotrophic factor increases in the uninjured dorsal root ganglion neurons in selective spinal nerve ligation model, J. Neurosci. 21 (2001) 4891-4900.

[9] W. Caumo, A. Deitos, S. Carvalho, J. Leite, F. Carvalho, J.A. Dussán-Sarria, M. da G. Lopes Tarragó, A. Souza, I.L. da S. Torres, F. Fregni, Motor cortex excitability and BDNF levels in chronic musculoskeletal pain according to structural pathology, Front. Hum. Neurosci. 10 (2016) 1-15.

[10] S.A. Zanette, J.A. Dussan-Sarria, A. Souza, A. Deitos, I.L.S. Torres, W. Caumo, Higher serum S100B and BDNF levels are correlated with a lower pressure-pain threshold in fibromyalgia, Mol. Pain 10 (2014) 46.

[11] R. Bianchi, I. Giambanco, R. Donato, S100B/RAGE-dependent activation of microglia via NF-kB and AP-1: co-regulation of COX-2 expression by S100B, IL-1 $\beta$ and TNF- $\alpha$, Neurobiol. Aging 31 (2010) 665-677.

[12] H. Imbe, A. Kimura, T. Donishi, Y. Kaneoke, Effects of restraint stress on glial activity in the rostral ventromedial medulla, Neuroscience 241 (2013) 10-21.

[13] G.R. Palos, T.R. Mendoza, G.M. Mobley, S.B. Cantor, C.S. Cleeland, Asking the community about cutpoints used to describe mild, moderate, and severe pain, J. Pain 7 (2006) 49-56.

[14] G. Peat, E. Thomas, R. Duncan, L. Wood, E. Hay, P. Croft, Clinical classification criteria for knee osteoarthritis: performance in the general population and primary care, Ann. Rheum. Dis. 65 (2006) 1363-1367.

[15] G. Nunes, L.V. de Castro, B. Wageck, V. Kume, G.S. Chiesa, M. de Noronha, Traduções para a língua portuguesa de questionários que avaliam lesões de joelho, Acta Ortopédica Brasileira 21 (2013) 288-294.

[16] F. Wolfe, D.J. Clauw, M.-A. Fitzcharles, D.L. Goldenberg, W. Häuser, R.S. Katz P. Mease, A.S. Russell, I.J. Russell, J.B. Winfield, Fibromyalgia criteria and severity scales for clinical and epidemiological studies: a modification of the ACR preliminary diagnostic criteria for fibromyalgia, J. Rheumatol. 38 (2011) 1113-1122.

[17] Headache Classification Committee of the International Headache Society (IHS), The international classification of headache disorders, 3rd edition (beta version), Cephalalgia 33 (2013) 629-808.
[18] M.Á. Freire, V.L.M. de Figueiredo, A. Gomide, K. Jansen, R.A. da Silva, P.V. da S. Magalhães, F.P. Kapczinski, M.Á. Freire, V.L.M. de Figueiredo, A. Gomide, K. Jansen, R.A. da Silva, P.V. da S. Magalhães, F.P. Kapczinski, Hamilton Scale: study of the psychometric characteristics in a sample from Southern Brazil, J. Bras. Psiquiatr. 63 (2014) 281-289.

[19] M.H. Gomes-Oliveira, C. Gorenstein, F. Lotufo Neto, L.H. Andrade, Y.P. Wang, Validation of the brazilian portuguese version of the beck depression Inventory-II in a community sample, Braz. J. Psychiatry 34 (2012) 389-394.

[20] $\mathrm{H}$. White, A heteroskedasticity-consistent covariance matrix estimator and a direct test for heteroskedasticity, Econometrica 48 (1980) 817-838.

[21] A. Fumal, J. Schoenen, Tension-type headache: current research and clinical management, Lancet Neurol. 7 (2008) 70-83.

[22] D. Bezov, S. Ashina, R. Jensen, L. Bendtsen, Pain perception studies in tension-type headache, Headache J. Head Face Pain 51 (2011) 262-271.

[23] L. Bendtsen, Central sensitization in tension-type headache-possible pathophysiological mechanisms, Cephalalgia 20 (2000) 486-508.

[24] P. Sarzi-Puttini, F. Atzeni, M. Di Franco, D. Buskila, A. Alciati, C. Giacomelli, A. Rossi, L. Bazzichi, Dysfunctional syndromes and fibromyalgia: a 2012 critical digest, Clin. Exp. Rheumatol. 30 (2012) 143-151.

[25] W. Pan, W.A. Banks, M.B. Fasold, J. Bluth, A.J. Kastin, Transport of brain-derived neurotrophic factor across the blood-brain barrier, Neuropharmacology 37 (1998) 1553-1561.

[26] B.A. Bus, M.L. Molendijk, B.J.W.H. Penninx, J.K. Buitelaar, G. Kenis, J. Prickaerts, B.M. Elzinga, R.C.O. Voshaar, Determinants of serum brain-derived neurotrophic factor, Psychoneuroendocrinology 36 (2011) 228-239.

[27] J. Nijs, M. Meeus, J. Versijpt, M. Moens, I. Bos, K. Knaepen, R. Meeusen, Brainderived neurotrophic factor as a driving force behind neuroplasticity in neuropathic and central sensitization pain: a new therapeutic target? Expert Opin. Ther. Targets 19 (2015) 565-576.

[28] M. Chassot, J.A. Dussan-Sarria, F.C. Sehn, A. Deitos, A. de Souza, R. Vercelino, I.L. Torres, F. Fregni, W. Caumo, Electroacupuncture analgesia is associated with increased serum brain-derived neurotrophic factor in chronic tension-type headache: a randomized, sham controlled, crossover trial, BMC Complement. Altern. Med. 15 (2015) 144.

[29] J. Nijs, C. Paul van Wilgen, J. Van Oosterwijck, M. van Ittersum, M. Meeus, How to explain central sensitization to patients with 'unexplained' chronic musculoskeletal pain: practice guidelines, Man. Ther. 16 (2011) 413-418.

[30] M. da G.L. Tarragó, A. Deitos, A.P. Brietzke, R. Vercelino, I.L.S. Torres, F. Fregni, W. Caumo, Descending control of nociceptive processing in knee osteoarthritis is associated with intracortical disinhibition, Medicine (Baltimore) 95 (2016).

[31] S.H. Eitner Annet, O. Hofmann Gunther, Mechanisms of Osteoarthritic Pain, Studies in humans and experimental modelso title, Front. Mol. Neurosci. 10 (2017) 349.

[32] Y.-T. Jeon, B.-G. Kim, Y.H. Park, H.-M. Sohn, J. Kim, S.C. Kim, S.S. An, S. Kim, Postoperative cognitive changes after total knee arthroplasty under regional anesthesia, Medicine (Baltimore) 95 (2016).

[33] J.V. Aredo, K.J. Heyrana, B.I. Karp, J.P. Shah, P. Stratton, Relating chronic pelvic pain and endometriosis to signs of sensitization and myofascial pain and dysfunction, Semin. Reprod. Med. 35 (2017) 088-097.

[34] K.L. Szuhany, M. Bugatti, M.W. Otto, A meta-analytic review of the effects of exercise on brain-derived neurotrophic factor, J. Psychiatr. Res. 60 (2015) 56-64.

[35] L.C. Stefani, I.L. da S. Torres, I.C.C. de Souza, J.R. Rozisky, F. Fregni, W. Caumo, BDNF as an effect modifier for gender effects on pain thresholds in healthy subjects, Neurosci. Lett. 514 (2012) 62-66. 